\title{
Extracting curve-skeletons from digital shapes using occluding contours
}

\section{Marco Livesu \& Riccardo Scateni}

\section{The Visual Computer}

International Journal of Computer Graphics

ISSN 0178-2789

Vis Comput

DOI 10.1007/s00371-013-0855-8
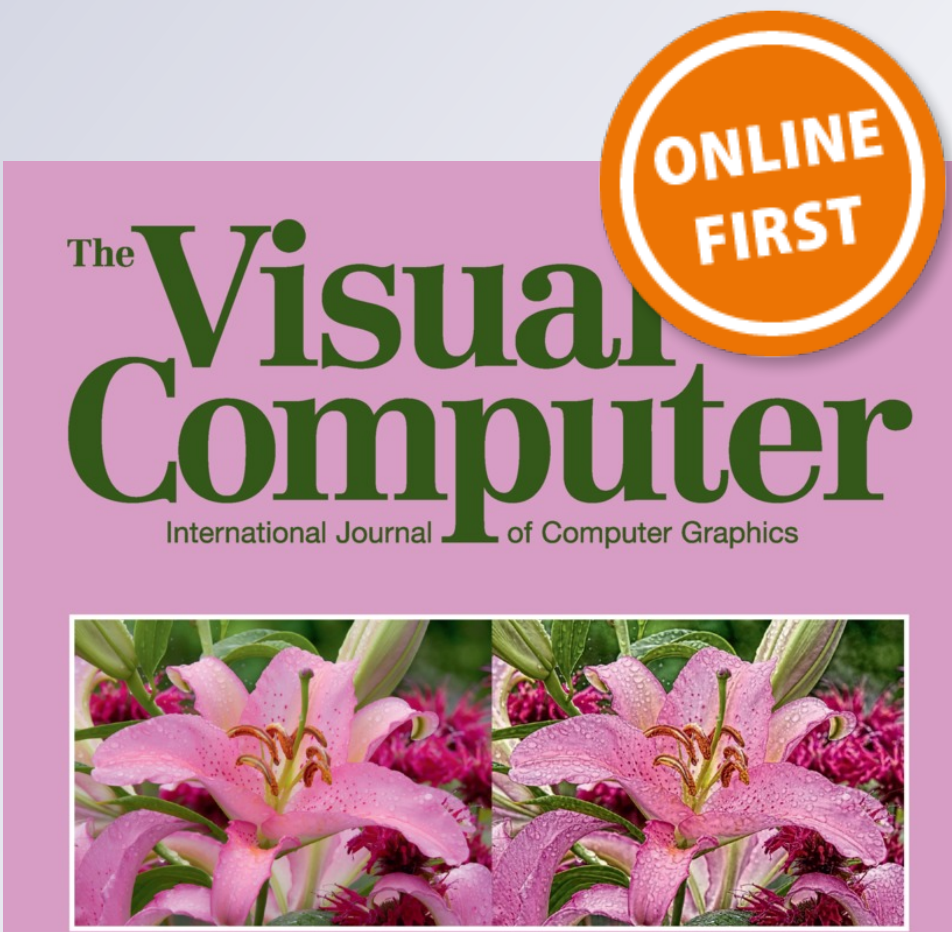

Special Issue: CGI'2013 Conference

Guest Editors and Conference Co-Chairs:

Qunsheng Peng

刑 Springer

\section{Springer}


Your article is protected by copyright and all rights are held exclusively by SpringerVerlag Berlin Heidelberg. This e-offprint is for personal use only and shall not be selfarchived in electronic repositories. If you wish to self-archive your article, please use the accepted manuscript version for posting on your own website. You may further deposit the accepted manuscript version in any repository, provided it is only made publicly available 12 months after official publication or later and provided acknowledgement is given to the original source of publication and a link is inserted to the published article on Springer's website. The link must be accompanied by the following text: "The final publication is available at link.springer.com". 


\title{
Extracting curve-skeletons from digital shapes using occluding contours
}

\author{
Marco Livesu $\cdot$ Riccardo Scateni
}

(C) Springer-Verlag Berlin Heidelberg 2013

\begin{abstract}
Curve-skeletons are compact and semantically relevant shape descriptors, able to summarise both topology and pose of a wide range of digital objects. Most of the stateof-the-art algorithms for their computation rely on the type of geometric primitives used and sampling frequency. In this paper, we introduce a formally sound and intuitive definition of a curve-skeleton, then we propose a novel method for skeleton extraction that relies on the visual appearance of the shapes. To achieve this result, we inspect the properties of occluding contours, showing how information about the symmetry axes of a 3D shape can be inferred from a small set of its planar projections. The proposed method is fast, insensitive to noise and resolution, capable of working with different shape representations, and easy to implement.
\end{abstract}

Keywords Curve-skeleton · Perceptual shape analysis

\section{Introduction}

Digital objects are flooding our environments: whether they are reproductions of real existing objects or produced by artists and designers, they are more and more complex bearing fine details. Skeletons and subdivisions in parts (segmentations) are compact and semantically sound approximations of the digital objects very useful in several different fields like, to name a few, computer animation, medical imaging, mechanical design, and shape archival, matching

M. Livesu $\cdot$ R. Scateni $(\bowtie)$

Dipartimento di Matematica e Informatica, University of Cagliari, via Ospedale, 72, 09124 Cagliari, Italy

e-mail: riccardo@unica.it

M. Livesu

e-mail: marco.livesu@unica.it and retrieval. Focusing on skeletons of three-dimensional objects, we can distinguish between surface-skeletons and curve-skeletons, where the former adhere more directly to the definition of collecting all the equidistant points from the boundaries of the shape, while the latter are collections of curves, and thus are, by definition, more compact and simple to manipulate than the former. Especially when dealing with the animation of digital characters, the curve-skeleton is an extremely important feature since it is the best guidance for changing the pose of the character and performing interpolations among poses.

Digital machineries (e.g. range scanners) are very good at capturing, even in the finer details, the surface of a shape, while human sight and perception are enormously more efficient in capturing the essentials of a shape: how many significant parts compose it, where the joints are, and so on. In other words, a good way to compute the curve-skeleton of a digital object is to try to mimic what the human mind does when looking at an object. This assumption is the basis of our approach as described in the rest of the paper.

\section{Related work}

Previous methods for curve-skeleton extraction can be sorted depending on the shape representation used as input. There are algorithms that are able to work on triangle meshes (e.g. $[3,8,11,27,28])$ which is the usual representation found in computer animation, others are well suited to process point clouds (e.g. [7, 29]) which range scanners produce as output with no further post-processing, and others with discrete volumes (e.g. $[10,12,17,31])$ since this is the format of acquisition for machinery like CAT and MRI. Most of these algorithms are able to perform well only when 


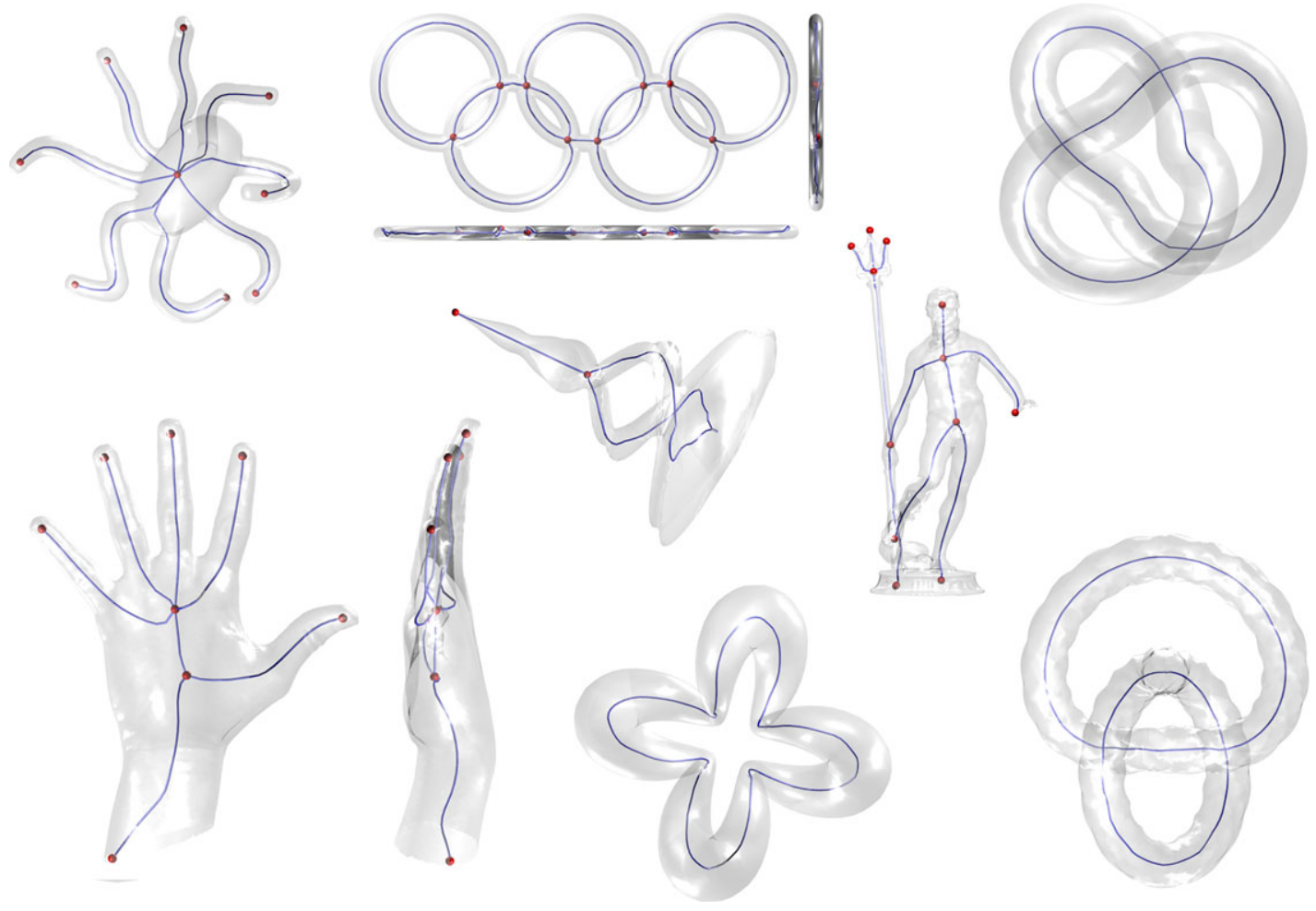

Fig. 1 Some results achieved with our method. The algorithm is able to perform with shapes of any genus, and with multiple connected components. The topology of the shape is preserved as long as the occlud-

the model resolution is fine enough, leading to unstable results when they are applied to coarse models. As skeletons are supposed to be high-level descriptors, the difference between the descriptors computed starting from high and low resolution models should be negligible. These are the biggest drawbacks of the state-of-the-art skeleton extraction algorithms: they are too tightly coupled with the geometric primitives and sampling frequency. They are working more on the machine side, focusing on primitives and resolution, than on the human side, focusing on appearance.

\subsection{Main contribution}

We give a formal definition of a curve-skeleton of digital objects built as unions of Generalised Cones. We extend the contour interpretation to partially occluded silhouettes, providing a definition of a locally unique symmetry point and moving the early visual perception theory from the global to the local setting. We formulate an algorithm to extract curve-skeletons from a set of occluding contours (Fig. 1).

\section{Theoretical background}

Shape analysis and recognition problems can be approached from another point of view, focusing more on appearance ing contours used to extract the skeletons carry enough information to observe it

and less on primitives. We largely based our work on the perception-based approach developed during the 1970s. People from M.I.T. Artificial Intelligence Laboratory, in particular David Marr, developed the theory of early visual perception, a study of how the human brain behaves while looking at an image containing the projection of a real object. We briefly recall the background of this theory with our contributions before entering into the details of our proposed algorithm for skeleton extraction.

\subsection{The generalised cones' theory}

According to the early perception theory, in [20] Marr and Nishihara stated that only the shapes belonging to the class of generalised cones can be fruitfully analysed. A generalised cone is the surface swept out by moving a crosssection of constant shape but smoothly varying size along an axis [4], or more formally

Definition 1 (Generalised cone) Let $\rho(r, \theta)$ be a simple closed planar curve twice continuously differentiable, and let $h$ be a twice continuously differentiable positive real function. Let $\Lambda$ be a line at some angle $\psi$ to the plane containing $\rho$, and denote positions along $\Lambda$ with $z$. Then, the surface $\mathcal{G C}=h \times \rho$ is a generalised cone with axis $\Lambda$, crosssection $\rho$, scaling function $h$, and eccentricity $\psi$. 


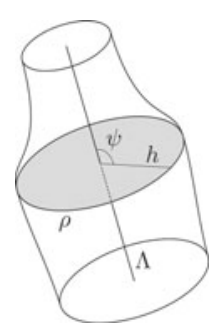

There are strong links between generalised cones and curve-skeletons. First of all, only objects that can be described in terms of generalised cones can be well described by curve-skeletons. It is really difficult, for instance, to imagine the skeleton of a mug, a door, or a crumpled newspaper because the natural axes of these shapes are either too weak to describe them or external to the shape. In [9], Cornea et al. suggested that the skeleton of a shape having a cavity should contain at least one loop around it but this would completely break down the topological connection between an object and its skeleton. To describe shapes containing tunnels or deep cavities, mono-dimensional shape descriptors like [21] or [22] would be no longer suitable.

We can express the relation between the generalised cones' primitives and curve-skeletons, formalising their descriptions. Given a real object $\mathcal{O}$,

$\mathcal{O}=\bigcup_{i=1}^{n} \mathcal{G C}_{i}\left(\Lambda_{i}, \rho_{i}, h_{i}, \psi_{i}\right)$

composed of $n$ generalised cones, according to the ideas expressed in [20], we define the skeleton of $\mathcal{O}$ as the union of the axes of each generalised cone, that is,

$\operatorname{Skel}(\mathcal{O})=\bigcup_{i=1}^{n} \Lambda_{i}$

Sticking to this definition of a skeleton, in the remainder of the paper, we will introduce a new algorithm for curveskeleton extraction that makes use of the tools provided by the early visual perception framework to catch the axes of the generalised cones composing a shape.

In [19], Marr formally proved that, under a few hypotheses, the axes $\Lambda_{i}$ of an object $\mathcal{O}$ may be found just by analysing one of its occluding contours (see [30]). A similar result, for a narrower set of shapes, has been achieved some years later by Rao and Medioni. In [24], they proved that the contour of a solid of revolution is symmetric about the projection of its axis for any view. These results can therefore be used to find the component axes of 3D objects without any a priori knowledge about their shapes.

The main drawbacks of the perceptual approach arise in the analysis of axes which are either foreshortened or hidden behind another part of the shape (i.e. occluded). To overcome the foreshortening problem, we decided to feed our algorithm with a set of silhouettes gathered from different points of view, making sure that every single axis is not foreshortened in at least one of them. To handle occlusions, we have moved Marr theory from the global to the local setting, so that we could to get as much information as possible from every single contour, either containing occlusions or not. The multi-view approach is also justified by the fact that shapes belonging to the class of unions of generalised cones can have complex topology or pose. For these shapes a view point that ensures that all the components are not occluded or foreshortened sometimes does not exists at all.

In the literature, there are several examples of centreline extraction from multiple views, especially in the Computer Vision field. Bullitt et al. [6] used stereo views to extract centrelines from medical datasets. Yoon et al. [32] employed a set of real cameras to catch many different silhouettes of a human, gather them together to compose a discrete volume, and then apply a curve-skeleton extraction algorithm based on Gradient Vector Flow. However, the theory of occluding contours has not ever been taken into account until recently. In a previous work [18] we assumed that the medial axes of a set of silhouettes of a 3D model were projections of the curve-skeleton of the shape. Then, we gathered the medial axis points in the discrete 3D space in order to reconstruct the skeleton with an ad hoc heuristic. Even though our assumption did not stand for every medial axis point of each silhouette, we have been able to achieve good results for a wide class of shapes of different topology and genus. Unfortunately, the drawbacks of our approach became evident when the number of occlusions was large. In these cases, the medial axis projected in the 3D space will bring a large amount of noise, making the skeleton extraction difficult and unstable. Nothing can be done to distinguish between noise and skeleton points because they both project into every single considered contour. Moreover, our method did not guarantee the skeleton paths to be centred because a discrete grid was used during the extraction and the smoothness was achieved only in post processing, thus deviating the curves from the medial lines of the shape. The method proposed in this paper is able to perform better with any kind of shape, producing incredibly noise-free point clouds (see Fig. 2) from which medial and naturally smooth skele-

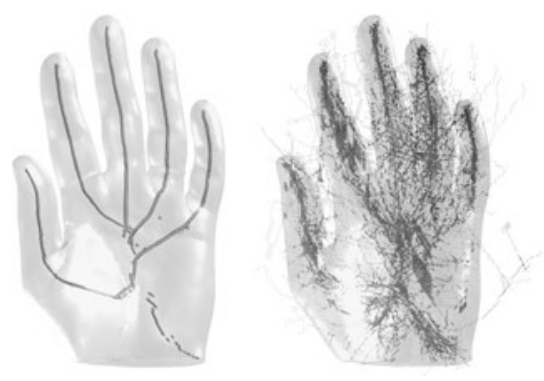

Fig. 2 Two raw point clouds produced by our method (left) and [18] (right) for the Olivier hand model. The same set of occluding contours has been used to make the comparison. By back-projecting only occlusion-free locally unique symmetry points, we can fully get rid of the noise, without any cleaning or post-processing. Moreover, working in the continuous setting, the skeleton paths suggested by the cloud are naturally smooth and appealing 
ton curves can be easily computed, without requiring any further post-processing.

The advantages of the perceptual approach are several. Firstly, it is usually faster than the state-of-the-art counterparts; secondly, it is completely unrelated with the geometric primitives used to describe the shape so that it is possible to extract coherent skeletons from any kind of representation (e.g. polygon meshes, implicit surfaces, parametric surfaces). Moreover, as long as the appearance of the object is preserved, resolution and noise has a negligible impact on the final result.

Most natural and artificial objects are unions of smooth elongated parts, and can thus be described as unions of generalised cone primitives.

\subsection{Inspecting symmetries}

The symmetry set of a domain $\Omega \in \mathbb{R}^{2}$ is the set of the centres of circles tangent to the boundary $\partial \Omega$ at least two distinct points. We here describe the Smoothed Local Symmetry, a local shape descriptor introduced in [5].

Let $a, b$ be two points

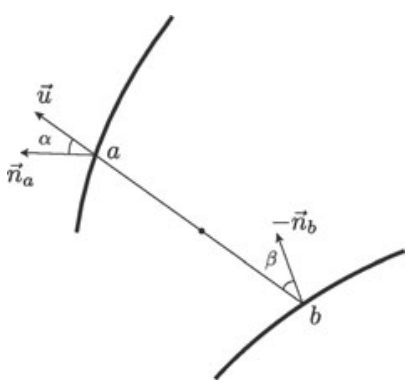
on the boundary $\partial \Omega$, and $\mathbf{u}$ the unit vector in the direction $\overline{a b}$. By definition, the midpoint of the segment $\overline{a b}$ belongs to $\operatorname{SLS}(\Omega)$ if and only if the angle $\alpha$ between $\mathbf{u}$ and the outward normal at $a$ is equal to the angle $\beta$ between $\mathbf{u}$ and the inward normal at $b$.

SLS is a very powerful shape descriptor as it is able to catch all the local symmetries of a contour; to have an example, one can look at Fig. 3, where a comparison with the Medial Axis Transform (MAT) is provided. This is particularly interesting because it makes the characterisation of locally unique symmetry points easier. A symmetry point $p=(a+b) / 2 \in \operatorname{SLS}(\Omega)$ is said to be locally unique if and only if

$\overline{a b} \cap \operatorname{SLS}(\Omega)=p$.

For example, looking at Fig. 3, one can note that most of the SLS points in the surroundings of the sharp corners of the boundary are not locally unique. When a point is locally unique, the distance $\|a-b\|$ can be used as an approximation of the local thickness of the shape. This particular property will be discussed in the remainder of the paper.

\subsection{Analysis of occluding contours}

When humans look at a silhouette, they perceive it as a particular 3D shape even though such silhouette could, in theory, be generated by an infinite number of shapes. In this

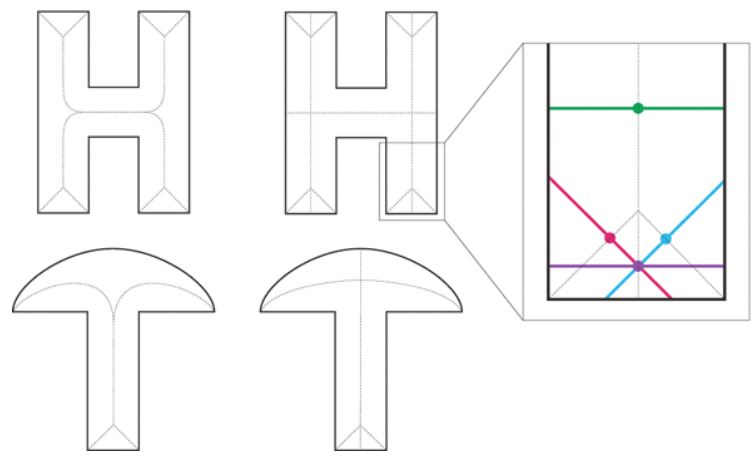

Fig. 3 A comparison between Medial Axis Transform (left) and Smoothed Local Symmetry (right). SLS is more powerful as it is able to capture all the symmetries of the figured shapes. Symmetry points are locally unique when their contact lines with the boundary do not intersect each other at any other symmetry point (close-up): red, blue and violet symmetry points are not unique, as their segments intersect each other, while the green point is locally unique

section, we will briefly introduce the theory of early visual perception, and we will discuss some improvements of it, useful to analyse occluded silhouettes.

Let $\mathcal{G C}$ be a generalised cone, and let $\Omega$ be its silhouette as seen from a viewpoint $v$, with $\pi_{v}$ being the linear projection which defines the mapping $\pi_{v}: \mathcal{G C} \rightarrow \Omega$. We call occluding contour the boundary $\partial \Omega$, and contour generator $\left(\mathcal{G C}_{\partial \Omega}\right)$ the set of points $p \in \mathcal{G C}$ that project onto $\partial \Omega$. In [19], Marr proved that, given a generalised cone $\mathcal{G C}(\Lambda, \rho, h, \psi)$ and a projection function $\pi_{v}$, if the axis of symmetry of the projection $\pi_{v}(\mathcal{G C})$ is unique, then it is the actual projection of the axis of symmetry $\Lambda$. To prove this, he assumed the following restrictions to be globally satisfied:

$R 1$ : Each point on the contour generator projects to a different point on the contour, that is, $\mathcal{G C}$ is convex as seen from $v$ or, in other words, the inverse $\pi_{v}^{-1}: \partial \Omega \rightarrow$ $\mathcal{G C}_{\partial \Omega}$ is one-valued;

$R 2$ : Nearby points on the contour arise from nearby points on the contour generator, that is, the mapping $\pi_{v}$ : $\mathcal{G C}_{\partial \Omega} \rightarrow \partial \Omega$ is continuous;

R3: The contour generator is planar.

The first thing we observed is that there is an interesting link between Marr's theory and the Smoothed Local Symmetry (SLS). When restrictions $R 1-R 3$ are satisfied, the following relation is satisfied as well

$\pi_{v}(\Lambda) \subseteq S L S(\Omega)$.

This is straightforward to prove because, by construction, each symmetry point in $\Omega$ belongs to $\operatorname{SLS}(\Omega)$. Therefore, if $\Lambda$ projects to the axis of symmetry of $\Omega$, it has to belong to $\operatorname{SLS}(\Omega)$. It is important to notice that, if the axis of symmetry of $\Omega$ is unique, then $\pi_{v}(\Lambda)=\operatorname{SLS}(\Omega)$. In any other case, at least one symmetry point $p$ such that $p \in \operatorname{SLS}(\Omega)$ 
and $p \notin \pi_{v}(\Lambda)$ must exist. One should note that the relation above is true only for medial descriptors able to catch every possible symmetry. For example, for the Medial Axis Transform (MAT) it would be false in the surroundings of the sharp corners of the shapes in Fig. 3.

What happens when the silhouette is partially occluded? Should we discard it? Or can we still get some good information from it? The first thing to do is to get rid of the symmetry points that have been affected by occlusions. Occlusion-free symmetry points can be locally characterised exploiting the locality properties of SLS. To do this, we define a function $\Phi: \Omega \rightarrow \mathbb{I}^{+}$that assigns to each point of the silhouette the number of points projected over it by $\pi_{v}$. Since each symmetry point $p=(a+b) / 2 \in \operatorname{SLS}(\Omega)$ depends only on the behaviour of the boundary restricted to $a$ and $b$, we can state that $p$ is occlusion-free if and only if each point in $\overline{a b}$ is occlusion-free, that is,

$\forall q \in \overline{a b}, \quad \Phi(q) \leq 2$.

Restrictions $R 1-R 3$ can be formulated in local fashion too, so now we are ready to define an occlusion-aware equivalent of the Marr's theory. Let $p=(a+b) / 2$ be an inner point of $\operatorname{SLS}(\Omega)$, if the symmetry is locally unique and occlusion-free in $p$, and if restrictions $R I-R 3$ locally hold, then

$p \in \pi(\Lambda)$.

Firstly, if $R 1-R 3$ are satisfied in $a$ and $b$ then $p$ will always be an SLS point, regardless of the behaviour of the rest of the boundary $\partial \Omega$. Secondly, let $\Omega_{1}, \Omega_{2}$ be the connected components of $\Omega \backslash \overline{a b}$. By construction, SLS is connected, hence two points $\lambda_{1} \in \Omega_{1}, \lambda_{2} \in \Omega_{2}$ always exist. Moreover, as the axis $\Lambda$ and the mapping $\pi$ are linear, $\pi(\Lambda)$ and $\overline{a b}$ have exactly one intersection. Therefore, due to (1) and (2), they intersect in $p$.

\section{Curve-skeleton extraction}

We propose here a novel curve-skeleton extraction algorithm that exploits the theory presented in the previous section to extract curve-skeletons of a 3D shape just by looking at multiple silhouettes of it. The idea is, at a high-level, very simple and intuitive. Firstly, we gather together multiple occluding contours of a 3D shape as seen from different viewpoints. Secondly, we extract from each contour the symmetry points that are projection of the axes of the generalised cones composing the object. We eventually match such symmetry points among the collected views in order to discover the axes in the 3D space using basic computer vision tools.

\subsection{Silhouette analysis}

The usage of a local analysis of the occluding contours is the major innovation of this paper and also the most innovative part of the proposed method over our approach previously presented in [18]. Basically, it consists in taking silhouettes, calculating their symmetries, and filtering all the symmetry points that are not unique or occlusion-free. To compute the SLS, an algorithm is proposed in [5]. While uniqueness can be easily checked during the SLS calculation, to check if a point is occlusion-free or not, a discrete version of the function $\Phi$ must be implemented. To do this, we employed the OpenGL stencil buffer, with the following setting:

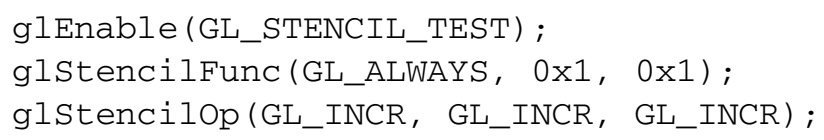

The code above makes sure that each time an object's primitive is projected over one pixel, the stencil buffer entry corresponding to it will be increased by one.

\subsection{View collection}

The choice of the viewpoints is the core factor in the construction of the perceptual skeleton of the object. In [14], Laurentini stated that the number of silhouettes necessary to optimally describe a polyhedron with $n$ faces is: unbounded if the viewpoints are not allowed to lie inside the convexhull of the object; $O\left(n^{5}\right)$ if the viewpoints are allowed to stay in the convex hull. Moreover, even if the view choice is optimal, some problems may occur, for example, in case the object contains cavities (see [13]). In our method, silhouettes are gathered just by rotating along the most important axis given by the Principal Component Analysis (PCA) of the $3 \mathrm{D}$ shape with a step of $3^{\circ}$. We used at most 60 silhouettes for complex models, thus covering a rotation of $180^{\circ}$ around the object (e.g. fertility and knots) and fewer views for simple models (e.g. Olympics). This choice proved to be sufficient in most of our experiments. However, the method does not depend on the particular camera positioning. Some heuristics, like [23, 25] and [26], would accommodate better contours for some shapes.

\subsection{Scanline matching}

In all our experiments, we used parallel projections in order to produce rectified sequences of contours. This choice makes the point matching problem very easy to solve because candidate matches always lie in the same scanline.

We used a two-step matching algorithm. Let $p^{(i)}$ and $p^{(j)}$ be two symmetry points belonging respectively to the $i$ th and $j$ th occluding contours. To have a positive match, $p^{(i)}$ and $p^{(j)}$ must belong to adjacent views, lie in the same scanline, and their distance along the scanline must be lower than 


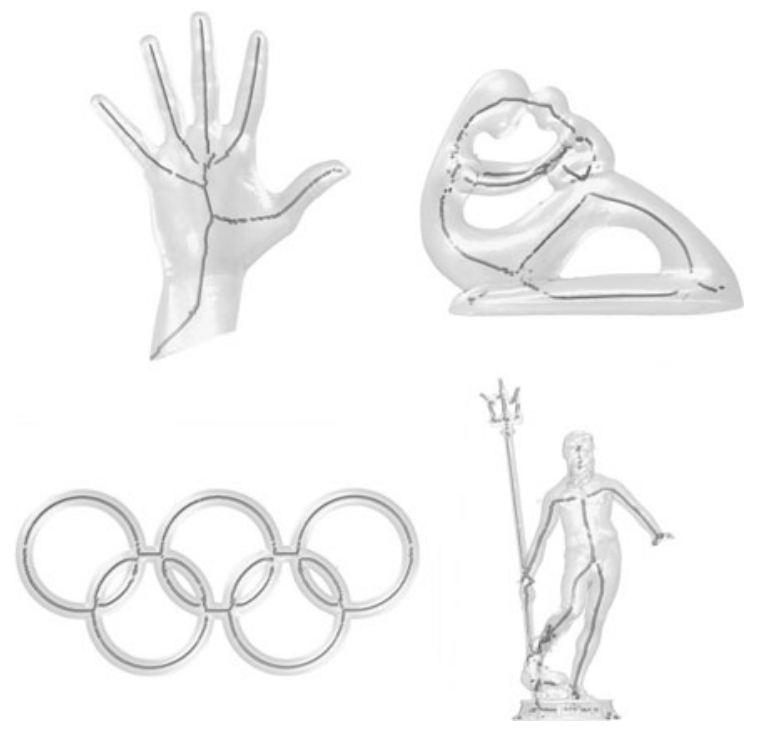

Fig. 4 Four raw point clouds produced back-projecting 2D symmetry points with our method. For each model we used a set of contours gathered by rotating around the most important axis given by the PCA. From each silhouette we select only the occlusion-free medial points, thus producing incredibly noise-free clouds for many different kinds of objects

a predefined threshold. In our simplified environment, this can be stated as

$|i-j|=1 \wedge p_{r}^{(i)}=p_{r}^{(j)} \wedge\left|p_{c}^{(i)}-p_{c}^{(j)}\right| \leq \delta$,

where $\delta$ is the maximum allowed displacement between two consecutive observations of the same point of a generalised cones axis (in all our tests, $\delta=2$ pixels). This first matching has the effect of grouping together all the subsequent observations of a symmetry point. We refer to this sets as bundles. However, the same point can be observed for a certain number of views, disappear due to an occlusion, and then appear again. In the second matching step, we merge together all the bundles that have been generated by projections of the same skeleton point. To do this, we apply the correspondence search algorithm proposed in [16]. Grouping in a single set all the observations of a skeleton point makes the algorithm more robust and also decreases the overall size and redundancy of the cloud (see Fig. 4).

\subsection{Back-projection}

After all the 2D symmetry point projections have been grouped in coherent bundles, the next step consists in projecting the points back to the shape space, in order to discover the skeleton paths. Every symmetry point in the bundle defines a projective ray in the space; the intersection of all these lines defines a candidate skeleton point in $\mathbb{R}^{3}$. A projective ray is a line and it can be expressed as the intersection of two planes in the space. Let $p \in \mathbb{R}^{3}$ be the coordinates of a general point belonging to the ray, and $\mathbf{d}$ be the
Direction Of Projection (DOP) of that ray. We can express it with the following linear system

$\left\{\begin{array}{l}\mathbf{d}_{y} x-\mathbf{d}_{x} y=\mathbf{d}_{y} p_{x}-\mathbf{d}_{x} p_{y}, \\ \mathbf{d}_{z} x-\mathbf{d}_{x} z=\mathbf{d}_{z} p_{x}-\mathbf{d}_{x} p_{y} .\end{array}\right.$

For any bundle we therefore set up a dense linear system $A \mathbf{x}=\mathbf{b}$ composed of $2 n$ equations, where $n$ is the number of symmetry points in the bundle. The matrix $A$ contains the directions of projection of the rays generating the symmetry points, the unknowns of the problem are the $x y z$ coordinates of a generalised cones' axes point. To increase the overall robustness, we discard bundles having fewer than 3 points; therefore, the system is always overdetermined and can be solved in the least squares sense, according to the normal equations $\tilde{\mathbf{x}}=\left(A^{T} A\right)^{-1} A^{T} \mathbf{b}$, such that

$\tilde{\mathbf{x}}=\arg \min _{\mathbf{x}}\|\mathbf{b}-A \mathbf{x}\|^{2}$.

An example of the point clouds produced by our method can be seen in Fig. 4. As one can note, due to the power of the SLS filtering and the robustness of the scanline matching, even for complex models like Fertility and Neptune, the clouds are almost noise-free and the skeleton paths are quite clean.

\subsection{Shape thickness}

Besides the $x y z$ coordinates of the skeleton points, another important information can be inferred from the SLS of the occluding contours. As stated in Sect. 3.2, when a symmetry point is unique, the distance from its closest contact points can be thought as an approximation of the local thickness of the shape as seen from a viewpoint. For each skeleton point we therefore set the local thickness as the lower distance observed among the SLS points involved in the backprojection step. This information will be really useful in the following step, where the skeleton paths will be reconstructed starting from the point cloud just created. Thickness information can also be used in a lot of applications. For example, in collision detection, where a coarse representation of a model can be really useful to detect collisions between articulated objects, drastically reducing the complexity of the problem. In Fig. 7, an example of reconstruction achieved using about 50 maximal balls has been produced for Fertility and Olivier hand models.

\subsection{Curve extraction}

The last step of our algorithm consists in the creation of the skeleton paths starting from the point cloud produced at the previous step. To do this, we used an approach first proposed in [15], and also used in [29]. As can be noted in Fig. 4, point clouds have a very thin structure along the branches, while points are a bit scattered near the joints. We 


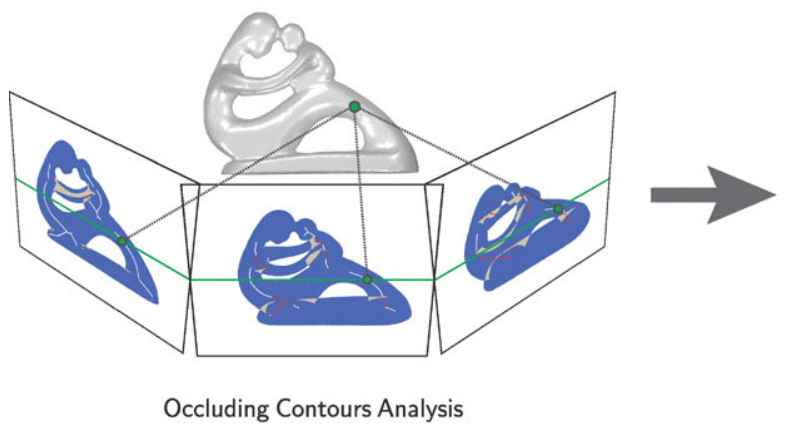

Fig. 5 The pipeline of the whole process: we first collect a set of occluding contours from neighbouring viewpoints; the result is a cloud of points sampling the axes of the generalised cones composing the shape;

then employ a 1D moving least squares (MLS) approach for the branches, iteratively projecting points onto their corresponding locally best fitting lines via principal component analysis (PCA). At each iterative step, we select a subset of points lying in a small neighbourhood. Since the cloud has been produced by the discrete representation of the contours, we use as neighbourhood size a quantity proportional to the distance between two points that would project to two adjacent pixels. However, as near joints points are too scattered, this neighbourhood measure is not able to work properly to infer branch connectivity. To thin the cloud and reveal how the branches connect with each other, we therefore apply Laplacian smoothing, using our estimation of the local thickness of the shape to infer point connectivity. It is now clear how important thickness is: in [29], the authors retrieved point connectivity with the help of the Mahalanobis distance. This was the most time consuming task of their algorithm; for $10 \mathrm{~K}$ points they needed about $3 \mathrm{~min}$ of computations. The pipeline of the whole process is sketched in Fig. 5.

\section{Results and comparisons}

We implemented our methods in $\mathrm{C}++$, using [2] for the manipulation of geometric data structures and [1] for numerical computations. Experiments were run on an iMac equipped with $2.66 \mathrm{GHz}$ Intel Core $2 \mathrm{Duo}$ and $4 \mathrm{~GB}$ RAM. The application runs on a single core. We used silhouettes of size $500 \times 500$ in all our tests; this choice proved to be a good trade-off between efficiency and precision. If necessary, higher resolution would permit higher precision.

We discuss here the skeletons produced with our algorithm according to the properties listed in [9]. Homotopy is observed but not guaranteed. It depends on the considered contours; we can guarantee it as long as any cone axis is projected without occlusions enough times to generate a bundle. Centredness is also observed but not guaranteed, in

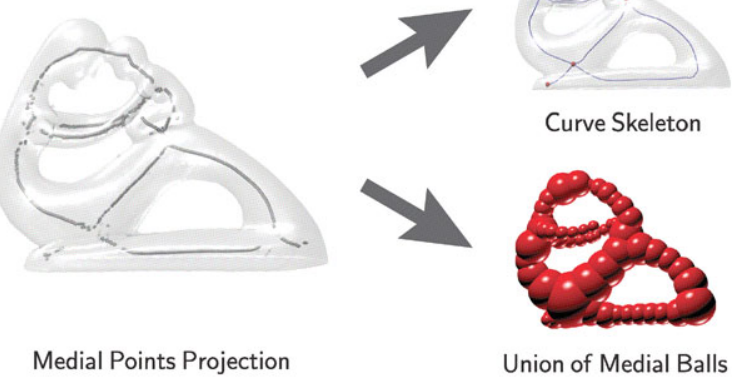

we eventually reconstruct the curve-skeleton, and an approximation of the shape is given by the union of the medial balls

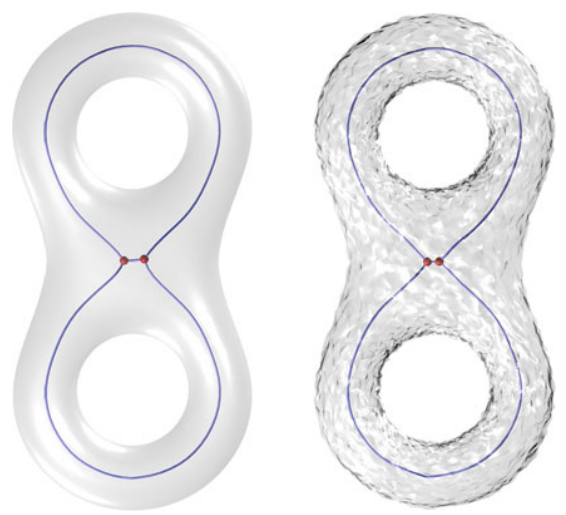

Fig. 6 Being based on shape appearance rather than on geometry, our method is able to handle noise successfully. In this image, two curve skeletons are extracted respectively from a noise-free and a noise-affected double torus

the sense that the least squares solution may move some skeleton point slightly further from the cone's axis. Being based on the visual appearance of the objects, the algorithm is very robust. It is almost insensitive to noise and missing parts (see Fig. 6). Moreover, thickness information allows a rough reconstruction of the shape (see Fig. 7).

In Table 1, we compared our method with three state-ofthe-art algorithms. In this comparison, we considered three synthetic shapes (a double torus and two knot models) with convex cross-section everywhere. We evenly sampled the skeletons and, for each skeleton point, we cut the mesh with a plane centred at it and having as normal direction the direction normal to the skeleton curve. We then measured the distance between the skeleton point and the centroid of the cross-section, normalising it with respect to the diagonal of the axis aligned bounding box containing the shape. As can be noted from the table, our results are comparable with the results achieved in [11] and [28] while [18] behaves slightly worse, probably because the curves are extracted from a voxel grid and then smoothed in post processing, thus deviating from the middle of the shape. 

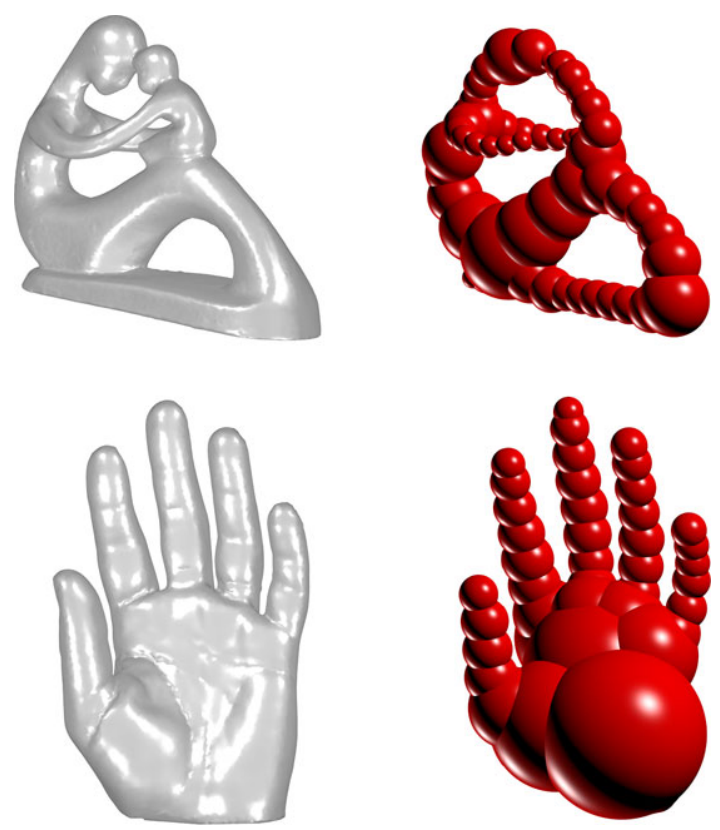

Fig. 7 Shape approximation of the Fertility model (55 balls) and Olivier Hand model (42 balls). Maximal balls are spanned along the skeleton paths, creating a good approximation of the original shape. This representation can be useful for applications like hole filling, surface reconstruction, and collision detection

Table 1 Numerical comparisons with three state-of-the-art solemnisation algorithms. We considered a set of synthetic shapes with convex cross-section. We first sub-sampled the skeletons and then, for each sample, measured its distance from the centroid of the local crosssection. Mean displacements are normalised with respect to the diagonal of the axis aligned bounding box containing the shape

\begin{tabular}{lccc}
\hline Method & Model & Avg displ. & Std. dev. \\
\hline Our method & Eight & 0.059918 & 0.009305 \\
Dey and Sun [11] $(\theta=0.0)$ & & 0.063134 & 0.012655 \\
Dey and Sun [11] $(\theta=0.5)$ & & 0.065679 & 0.012750 \\
Tagliasacchi et al. [28] & & 0.001762 & 0.011715 \\
Livesu et al. [18] & & 0.065188 & 0.025373 \\
Our method & Knot 1 & 0.044990 & 0.022698 \\
Dey and Sun [11] $(\theta=0.0)$ & & 0.046228 & 0.023781 \\
Dey and Sun [11] $(\theta=0.5)$ & & 0.050125 & 0.024415 \\
Tagliasacchi et al. [28] & & 0.001570 & 0.012924 \\
Livesu et al. [18] & & 0.092037 & 0.027562 \\
Our method & Knot 1 & 0.052322 & 0.008020 \\
Dey and Sun [11] $(\theta=0.0)$ & & 0.054011 & 0.008043 \\
Dey and Sun [11] $(\theta=0.5)$ & & 0.054063 & 0.008049 \\
Tagliasacchi et al. [28] & & 0.001742 & 0.013306 \\
Livesu et al. [18] & & 0.079337 & 0.018083 \\
\hline
\end{tabular}

In Table 2, we report running times and number of considered contours for some models we tested. Our method runs faster than the state-of-the-art counterparts, especially for high resolution models. For instance, on models with approximatively 60,000 faces, we compute the skeleton in half a second, [28] takes a few seconds, [3] a few minutes, and [11] almost half an hour. Moreover, since the most timeconsuming task is the rasterisation, times may be further lowered using smart rendering techniques.

\subsection{Implicit surfaces}

Triangle meshes are the most common data structure for surface representation, and all the state-of-the-art algorithms we compared with are able to deal with them. However, they have two big drawbacks: they are only $\mathcal{C}^{0}$-continuous and their topology must be checked explicitly in order to avoid self-intersections that are not found in real world objects.

When one wants to over-

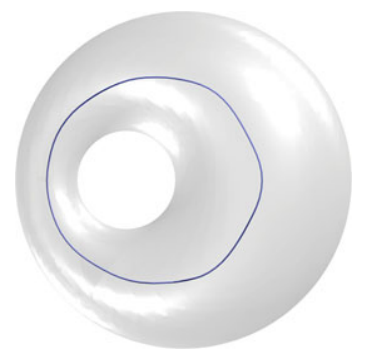
come these drawbacks, one uses other surface representations like parametric or implicit surfaces. One of the greatest advantages of our method (as well as [18]) is the possibility to deal with this representations without any additional effort: the computation of the silhouettes can be merely reduced to the computation of the projection of the surface onto the projection planes. Meshing an implicit surface is instead a time consuming task, and this is why the surface is usually calculated on-the-fly at raster time, without any explicit representation. To the best of our knowledge, contour-based approaches are the only techniques available in the literature for the direct solemnisation of implicit surfaces. In the figure above, we show an example of the curve skeleton extracted from a Dupin's cyclide with equation $693 x^{4}+1386 x^{2} y^{2}-18880 x^{2} y+1386 x^{2} z^{2}+59000 x^{2}+$ $693 y^{4}-18880 y^{3}+1386 y^{2} z^{2}+187000 y^{2}-18880 y z^{2}-$ $800000 y+693 z^{4}+67000 z^{2}+1250000=0$. To compute the projections, raster the silhouettes, and, finally, compute the skeleton, we considered the zero set of the equation.

\section{Limitations and further work}

It is worth to clearly remind that the method we describe here is meant to work only for objects that can be represented by a union of a finite number of generalised joined cones. To describe shapes with weakly defined symmetry axes, like mugs, busts or buildings, one should probably use a different descriptor.

The multi-view based approach, besides the advantages, carries some limitations. Back-projected points are not guaranteed to be inside the shape. Even if the matching algorithm is extremely robust, it can happen that when there are 
Table 2 Time splitting (in milliseconds) of the skeleton extraction pipeline

\begin{tabular}{lrrrrr}
\hline Model & Faces & $\begin{array}{l}\text { Occluding } \\
\text { contours }\end{array}$ & $\begin{array}{l}\text { Rasterisation } \\
\text { time }(\mathrm{ms})\end{array}$ & $\begin{array}{l}\text { Skeletonisation } \\
\text { time }(\mathrm{ms})\end{array}$ & $\begin{array}{l}\text { Total } \\
\text { time (ms) }\end{array}$ \\
\hline Olivier Hand & 49,586 & 37 & 646 & 249 & 895 \\
Hand & 273,060 & 6 & 247 & 74 & 321 \\
Neptune & 56,112 & 32 & 321 & 250 & 571 \\
Double torus & 1,536 & 6 & 22 & 79 & 101 \\
Fertility & 50,000 & 60 & 546 & 391 & 937 \\
Twirl & 10,402 & 20 & 112 & 175 & 287 \\
Knot \#1 & 4,160 & 60 & 202 & 382 & 584 \\
Knot \#2 & 6,400 & 60 & 227 & 396 & 623 \\
Knot \#3 & 11,520 & 60 & 266 & 302 & 568 \\
Olympics & 7,862 & 6 & 24 & 155 & 179 \\
Spider & 23,808 & 60 & 357 & 442 & 799 \\
\hline
\end{tabular}

a lot of occlusions some points may be projected outside the shape due to a wrong matching. Moreover, as we are dealing with contours, we can only guarantee that the skeleton lies inside the Visual Hull of the object rather than the object itself. We lack a stop rule for the silhouette acquisition system. Our method uses a set of silhouettes constructed by rotating around the most important PCA direction; however, as one can note by looking at Table 2, some models need few contours and some others need more. A stop rule to automatically determine how many views we need should be used. Regarding shape representations, our method is able to work with any polygonal mesh and any discrete volume representation. Any kind of representation that allows the calculation of occlusions can be used interchangeably. We are currently working to extend the work to point clouds. This can be done by inferring the occlusion map (the discretized $\Phi$ function) by post processing the information contained in the stencil buffer. We are also working to extend this 2D-to$3 \mathrm{D}$ paradigm to real objects moving in front of a camera or multiple views of a static object.

\section{Conclusions}

In this paper, we introduced an intuitive definition of a curve-skeleton and we showed how the axes of a complex generalised cone can be inferred just by looking at their planar projections from a few viewpoints. We extended the classical theory of early visual perception in order to deal with occlusions, proposing a solemnisation algorithm which is fast, insensitive to noise and missing data, and easy to implement. Moreover, we showed that the results produced by our algorithm are qualitatively comparable with the stateof-the-art counterparts, but the algorithm is more versatile because it can be used with any kind of surface representation. We also showed that the centredness of the skeletons produced by our algorithm is higher than previous contourbased approaches because our skeleton curves are naturally smooth and do not need any post processing.

\section{References}

1. http://eigen.tuxfamily.org/

2. http://vcg.sourceforge.net/

3. Au, O.K.C., Tai, C.L., Chu, H.K., Cohen-Or, D., Lee, T.Y.: Skeleton extraction by mesh contraction. ACM Trans. Graph. 27, 44:144:10 (2008)

4. Binford, T.O.: Visual perception by computer. In: IEEE Conference on Systems and Control, vol. 261, p. 262 (1971)

5. Brady, M., Asada, H.: Smoothed local symmetries and their implementation. Int. J. Robot. Res. 3(3), 36-61 (1984)

6. Bullitt, E., Liu, A., Pizer, S.: Three-dimensional reconstruction of curves from pairs of projection views in the presence of error. I. Algorithms. Med. Phys. 24, 1671-1678 (1997)

7. Cao, J., Tagliasacchi, A., Olson, M., Zhang, H., Su, Z.: Point cloud skeletons via Laplacian based contraction. In: Shape Modeling International Conference (SMI) 2010, pp. 187-197. IEEE Press, New York (2010)

8. Chuang, M., Kazhdan, M.: Fast mean-curvature flow via finiteelements tracking. Comput. Graph. Forum 30, 1750-1760 (2011)

9. Cornea, N., Silver, D., Yuan, X., Balasubramanian, R.: Computing hierarchical curve-skeletons of 3D objects. Vis. Comput. 21(11), 945-955 (2005)

10. Cornea, N.D., Silver, D., Min, P.: Curve-skeleton applications. In: Proc. of IEEE Visualization, pp. 95-102 (2005)

11. Dey, T.K., Sun, J.: Defining and computing curve-skeletons with medial geodesic function. In: Proceedings of the fourth Eurographics Symposium on Geometry processing (SGP '06), Airela-Ville, Switzerland, pp. 143-152. Eurographics (2006)

12. Hassouna, M.S., Farag, A.A.: Robust centerline extraction framework using level sets. In: CVPR '05, vol. 1, pp. 458-465 (2005)

13. Laurentini, A.: How far 3D shapes can be understood from $2 \mathrm{D}$ silhouettes. IEEE Trans. Pattern Anal. Mach. Intell. 17(2), 188195 (1995)

14. Laurentini, A.: How many $2 \mathrm{D}$ silhouettes does it take to reconstruct a 3D object? Comput. Vis. Image Underst. 67(1), 81-87 (1997)

15. Lee, I.K.: Curve reconstruction from unorganized points. Comput. Aided Geom. Des. 17(2), 161-177 (2000) 
16. Leordeanu, M., Hebert, M.: A spectral technique for correspondence problems using pairwise constraints. In: Proceedings of the Tenth IEEE International Conference on Computer Vision (ICCV '05), vol. 2, pp. 1482-1489. IEEE Computer Society, Washington (2005)

17. Liu, L., Chambers, E.W., Letscher, D., Ju, T.: A simple and robust thinning algorithm on cell complexes. Comput. Graph. Forum 29(7), 2253-2260 (2010)

18. Livesu, M., Guggeri, F., Scateni, R.: Reconstructing the curveskeletons of 3D shapes using the visual hull. IEEE Trans. Vis. Comput. Graph. 18(11), 1891-1901 (2012)

19. Marr, D.: Analysis of Occluding Contour. Proc. R. Soc. Lond. B, Biol. Sci. 197(1129), 441-475 (1977)

20. Marr, D., Nishihara, H.K.: Representation and Recognition of the Spatial Organization of Three-Dimensional Shapes. Proc. R. Soc. Lond. 200(1140), 269-294 (1978)

21. Martin, T., Chen, G., Musuvathy, S., Cohen, E., Hansen, C.: Generalised swept mid-structure for polygonal models. Comput. Graph. Forum 31, 805-814 (2012)

22. Miklos, B., Giesen, J., Pauly, M.: Discrete scale axis representations for 3D geometry. ACM Trans. Graph. 29, 101:1-101:10 (2010)

23. Pujari, A.: Volume intersection for shape from silhouettes. Sadhana 18, 325-336 (1993)

24. Rao, K., Medioni, G.: Useful geometric properties of the generalised cone. In: Computer Society Conference on Proceedings of Computer Vision and Pattern Recognition 1988 (CVPR '88), pp. 276-281 (1988)

25. Secord, A., Lu, J., Finkelstein, A., Singh, M., Nealen, A.: Perceptual models of viewpoint preference. ACM Trans. Graph. 30(5), 109:1-109:12 (2011)

26. Shanmukh, K., Pujari, A.K.: Volume intersection with optimal set of directions. Pattern Recogn. Lett. 12(3), 165-170 (1991)

27. Sharf, A., Lewiner, T., Shamir, A., Kobbelt, L.: On-the-fly curveskeleton computation for 3D shapes. Comput. Graph. Forum 26(3), 323-328 (2007)

28. Tagliasacchi, A., Alhashim, I., Olson, M., Zhang, H.: Mean curvature skeletons. Comput. Graph. Forum 31(5), 1735-1744 (2012)

29. Tagliasacchi, A., Zhang, H., Cohen-Or, D.: Curve skeleton extraction from incomplete point cloud. ACM Trans. Graph. 28(3), 1-9 (2009)
30. Waltz, D.: Understanding line drawings of scenes with shadows. In: Winston, P. (ed.) The Psychology of Computer Vision, pp. 1991. McGraw-Hill, New York (1975)

31. Wang, Y.S., Lee, T.Y.: Curve-skeleton extraction using iterative least squares optimization. IEEE Trans. Vis. Comput. Graph. 14(4), 926-936 (2008)

32. Yoon, S.M., Malerczyk, C., Graf, H.: 3D skeleton extraction from volume data based on normalized gradient vector flow. In: The 17th International Conference in Central Europe on Computer Graphics, Visualization and Computer Vision, Plzen, Czech Republic, pp. 177-182 (2009)

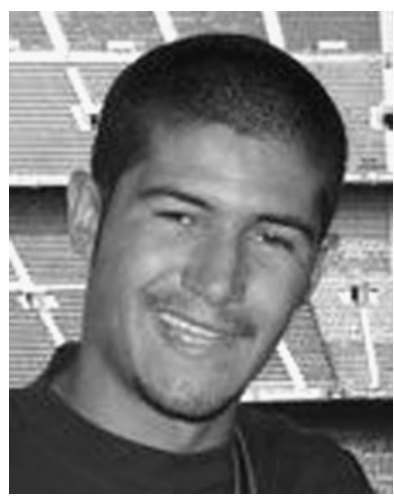

Marco Livesu received his Master's Degree Magna Cum Laude from the University of Cagliari, Italy, in September 2010. Currently, he is a $\mathrm{Ph} . \mathrm{D}$. student in the department of Computer Science at University of Cagliari. His research interests include computer graphics, shape description, shape deformation and computer vision.

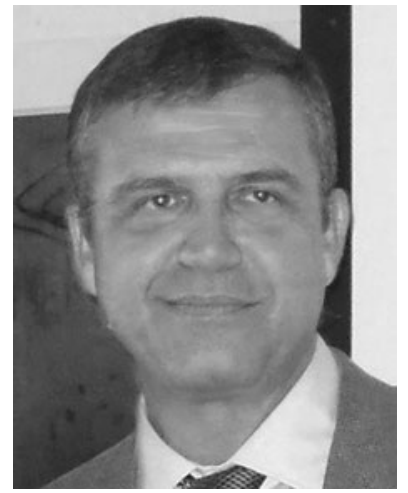

Riccardo Scateni is an Associate Professor at the Department of Mathematics and Computer Science of the University of Cagliari. He joined the University after a long period spent at CRS4 in Cagliari. He has worked in Scientific Visualization for a long period and his current research interests are in the field of Computer Graphics, especially Geometry Processing, and Interaction Design. 